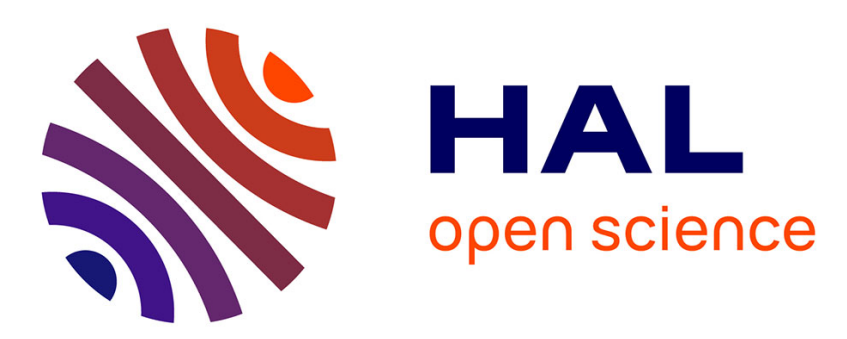

\title{
Lercoul (Pyrénées ariégeoises): un site sidérurgique du IIIe s. de notre ère
}

Claude Dubois

\section{To cite this version:}

Claude Dubois. Lercoul (Pyrénées ariégeoises): un site sidérurgique du IIIe s. de notre ère. Gallia - Archéologie de la France antique, 2000, Mines et métallurgies en Gaule, 57, pp.53-62. 10.3406/galia.2000.3209 . hal-01902596

\section{HAL Id: hal-01902596 https://hal.science/hal-01902596}

Submitted on 20 Jan 2020

HAL is a multi-disciplinary open access archive for the deposit and dissemination of scientific research documents, whether they are published or not. The documents may come from teaching and research institutions in France or abroad, or from public or private research centers.
L'archive ouverte pluridisciplinaire HAL, est destinée au dépôt et à la diffusion de documents scientifiques de niveau recherche, publiés ou non, émanant des établissements d'enseignement et de recherche français ou étrangers, des laboratoires publics ou privés.

\section{(ㅇ)(1) $\$$}

Distributed under a Creative Commons Attribution - NonCommercial - NoDerivatives $\mid 4.0$ 


\title{
LERCOUl (PyRÉNÉES ARIÉGEOISES)
}

\author{
Un site sidérurgique du III s. de notre ère
}

\author{
Claude DuboIS
}

Mots-clés. Bas fourneaux, schiste, scories, gethite, charbon de bois, ${ }^{14} \mathrm{C}$, superstructure.

Key-words. Shaft furnaces, schist, slags, gæthite, charcoal, ${ }^{14} C$, superstructure.

Résumé. À Lercoul, deux bas fourneaux jumeaux puis un bas fourneau isolé ont produit environ $400 \mathrm{t}$ de scories au cours du III $s$. de notre ère, à partir de geethite et oligiste riches en manganèse, et de charbon de bois de hêtre. Le creuset était encastré dans le socle en schiste de la montagne et la superstructure formant cheminée était bâtie en moellons de schiste liés à l'argile. Argile et schiste pilé constituaient le revêtement réfractaire interne. La paroi frontale permettait l'évacuation des scories coulées à sa base, dans un chenal, pour les deux fours jumeaux. Des fragments de tuyères indiquent l'emploi d'une soufflerie. Les éponges étaient épurées sur place, probablement après avoir été réchauffées. L'indigence du site en mobilier a nécessité le recours aux datations ${ }^{14} \mathrm{C}$.

\begin{abstract}
At Lercoul (Pyrenean mountains, Ariège, France), two tuin iron making shaft furnaces and a third one nearby produced about 400 tons of slag during the $3^{\text {rd }}$ century AD. The ore consisted of gothite and oligiste with manganese. It was reduced with charcoal from beech. The bottom was cut in the schist rock and the superstructure was made of slate blocks and clay. Inside the shaft was coated with clay and small pieces of slate mixed together. At the foot of the front wall, there was a lap-hole for lapping slag into a small channel. The discovery of pieces of broken tuyeres shows that bellows were used for blowing air into the furnace. The iron bloom was reheated and hammered on the spot for removal of the entrapped slag. Considering the scarcity of dated archaeological items, radiocarbon dating has been used.
\end{abstract}

\section{CONTEXTE}

Dans les Pyrénées ariégeoises et le bassin versant du Vicdessos, les pics de Ganchette ( $1596 \mathrm{~m}$ ) et de la Bède $(1685 \mathrm{~m})$ séparent la vallée de Siguer et le versant de Lercoul à l'est, de la vallée et du versant de Sem à l'ouest (fig. 19). Le premier des deux sommets marque aussi la limite entre la célèbre mine de fer médiévale et moderne de Rancié du côté de Sem, et un chapelet de mines de fer sur Lercoul. Ces minéralisations filoniennes à remobilisations karstiques locales étaient riches en hématite et gœthite dans une gangue quartzeuse. La roche encaissante est une dolomie dévonienne ${ }^{75}$. Une intercalation

75. C. Dubois, B. Ancel, le karst dans les mines de fer de Rancié et de Lercoul (Pyrénées ariégeoises), in: Karst et Mines, Actes du colloque, Paris, 28-29 mai 1994, éd. AEDEH, à paraitre. de schiste se situe au sud de cette formation, entre les deux pics. La forêt actuelle traduit cette structure géologique: tandis que la hêtraie couvre les terrains calcaires, une sapinière marque le socle schisteux. Cette futaie de sapins, mentionnée dans la Réformation forestière de 1669 , constitue une relique atypique dans le paysage ariégeois. En effet si la sapinière était la forêt climacique à la fin de la dernière glaciation, le réchauffement permit l'apparition du hêtre et celui-ci fut vivement favorisé par l'anthropisation à partir de l'âge du Bronze. Du XIV ${ }^{\mathrm{e}} \mathrm{s}$. au milieu du XIX' $\mathrm{X}$., presque toutes les forêts ariégeoises furent anéanties par la pression de la métallurgie au charbon de bois; seuls des taillis de hêtres subsistèrent ça et là et de rares futaies, parfois de sapins, dans les endroits protégés. À Lercoul, le toponyme de la Bède fait référence à une telle protection du 


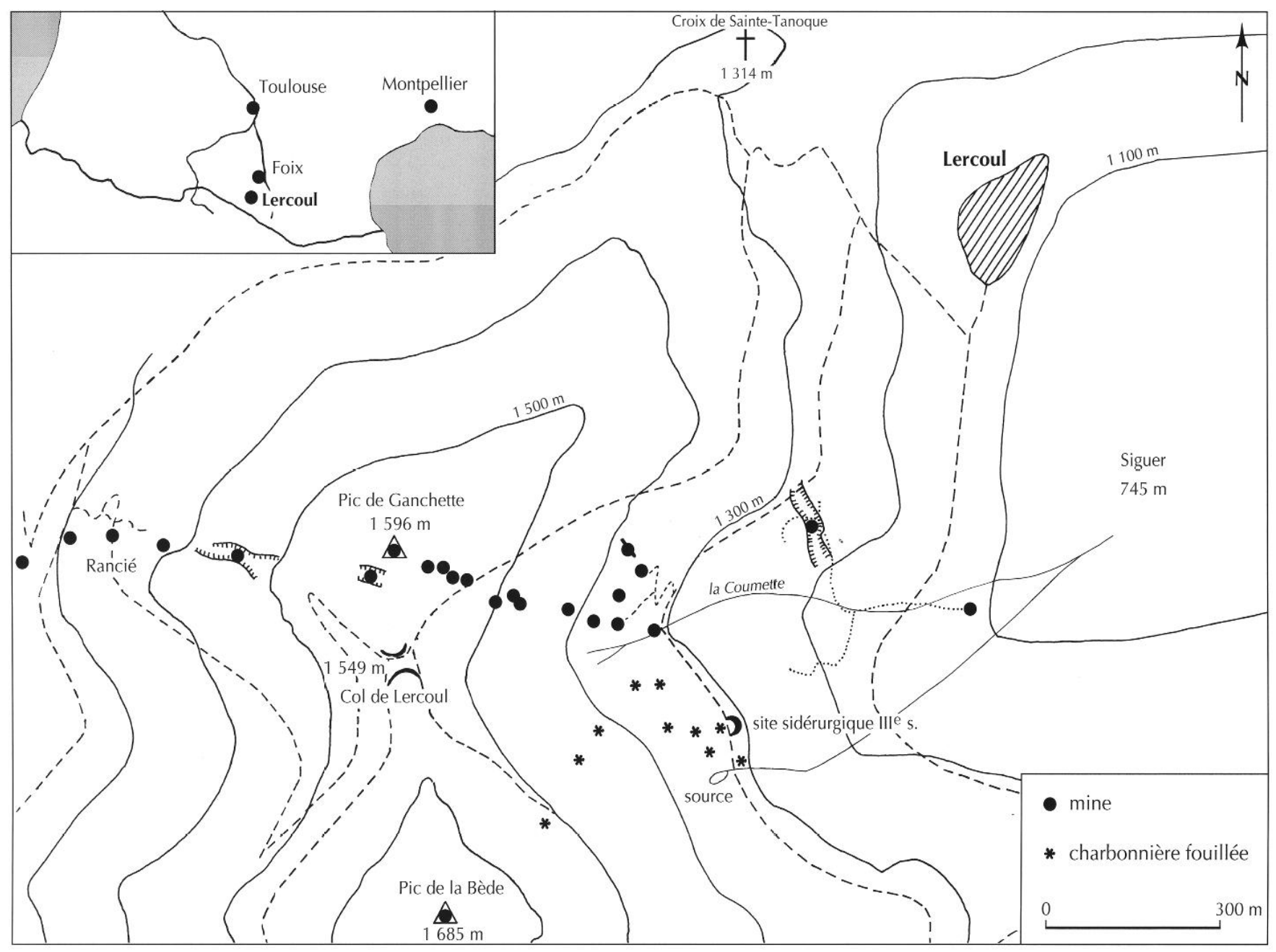

Fig. 19 - Lercoul (Ariège) : carle de localisation des vestiges.

versant: il provient de l'occitan débès, équivalent de "défens » en français ${ }^{76}$. La pente de la montagne est de $35^{\circ}$. Cette description du milieu ne saurait omettre la mention d'une source pérenne située à 1340 m d'altitude, dans une fracture du schiste.

Il paraît assuré qu'à l'époque romaine la haute Ariège faisait partie de la cité de Toulouse. Pour l'Antiquité et le début du Moyen Âge, l'archéologie locale est particulièrement pauvre ${ }^{77}$. Aucune découverte n'est répertoriée

76. H. Cavailles, La vie pastorale et agricole dans les Pyrénées des Gaves de l'Adour et des Nestes, Paris, éd. Colin, 1930, 413 p. Aujourd'hui la forêt royale de Lercoul est une forêt domaniale, géréc par l'Office national des forêts qui a été partenaire des fouilles archéologiques et que nous remercions.

77. J.-M. Escudé-Quillet, C. Maissant, L’Ariège, Carte archéologique de la Gaule, Paris, Maison des sciences de l'homme, 1997, p. 107 et 163. sur la commune de Lercoul. À Sem, quelques monnaies romaines auraient été découvertes, plus ou moins proches de la mine de Rancié, au XIX s. La vallée de Siguer est la voie la plus directe pour franchir la chaîne entre l'Ariège et l'Andorre. Elle communique avec la vallée d'El Serrat et d'Ordino par le Port Vieux de Siguer (alt. : $2436 \mathrm{~m}$ ), où des monnaies antiques auraient été découvertes également au siècle dernier. Les vallées voisines ne sont guère mieux loties et l'on pourrait croire, au vu des vestiges connus, qu'elles furent désertées entre le Paléolithique et le milieu du Moyen Âge. S'il est certain que la romanisation fut très discrète dans ces montagnes éloignées de tout centre administratif et économique, il est possible que la pauvreté du contexte archéologique soit aussi due à l'absence de recherche archéologique sur ces périodes historiques et probable- 
ment à une permanence de l'habitat traditionnel sur les sites favorables.

\section{LE SITE SIDÉRURGIQUE DU III ${ }^{\mathrm{e}} \mathrm{S}$.}

Le site sidérurgique du $\mathrm{III}^{c} \mathrm{~s}$. présenté ici fut révélé par la présence de scories dans le talus d'une piste forestière ouverte en contrebas, il y a une dizaine d'années. Il se trouve à $1330 \mathrm{~m}$ d'altitude, à quelque $300 \mathrm{~m}$ au sud-est des principales mines de fer de Lercoul (fig. 19).

Il s'agit d'une plate-forme qui interrompt la pente de la montagne sur $5 \mathrm{~m}$ de largeur maximale et $20 \mathrm{~m}$ de longueur en nord-sud (fig. 20). Des sapins plus que centenaires se dressaient sur cet espace. Un chemin longeait le site dans toute sa longueur à $2 \mathrm{~m}$ au-dessus de la plate-forme.

À l'origine, les métallurgistes ont peut-être tiré parti d'une petite rupture de pente, dans la mesure où le substrat schisteux forme un replat d'environ $3 \mathrm{~m}$ de large à l'endroit où les trois fourneaux furent établis (fig. 21 et 22). Ils ont décapé le rocher sur au moins $4 \mathrm{~m}$ de largeur en est-ouest, et l'accumulation des déblais de terre argileuse et de plaques de schiste a formé l'essentiel de la plate-forme. Par la suite, le rejet des scories dans la pente a contribué à élargir cet espace.

Le recouvrement de la partie nord du site se présente, globalement, comme une succession de lentilles de charbons de bois plus ou moins étendues et d'amas composés de scories, de plaques de schiste souvent rubéfiées, de nodules de quartz, de minerai de fer concassé et chauffé et de charbons de bois. Une nappe d'argile à cailloutis schisteux, présentant des éléments chauffés, s'intercale dans cette succession. Topographi-

Fig. 20 - Plan de synthèse du sile sidérurgique de Lercoul (Ariège) (III s.) : 1, dépôt de charbons de bois daté de 180-415 cal. AD; 2, limite supérieure du ferrier recouvrant l'éboulis du bas fourneau $A$; 3, fosse ouverte dans l'argile de la plate-forme; 4, limite supérieure certaine du ferrier daté de 70-320 cal. $A D$; $4^{\text {bis }}$, limite supérieure probable du ferrier daté de 70-320 cal. AD; 5, éboulis de la superstruclure du bas fourneau $A ; 6$, sol de travail du bas fourneau $A ; 7$, bas fourneau $A ; 8$, bas fourneau $B ; 9$, bas fourneau $C ; 10$, limite supérieure du ferrier dalé. de $180-420 \mathrm{cal}$. AD); 11, sol d'épuration contenant des battitures; 12 , foyer sud ; 13 , ferrier. quement, deux ensembles se distinguent dans le recouvrement de l'extrémité nord de la plate-forme. Les couches supérieures (fig. 20, $\mathrm{n}^{\text {os }} 1$ et 2) s'étalent sur la partie plane du site où elles scellent une fosse ovoïde

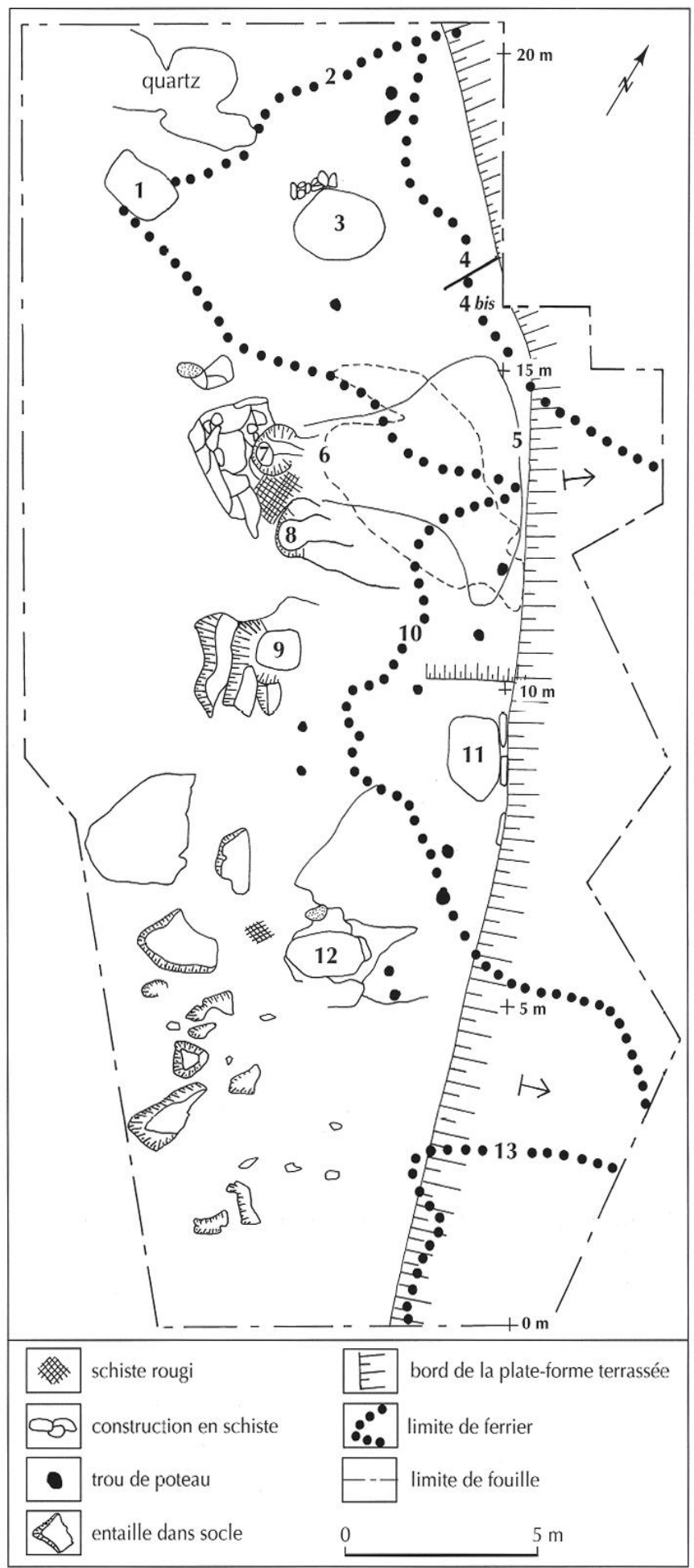




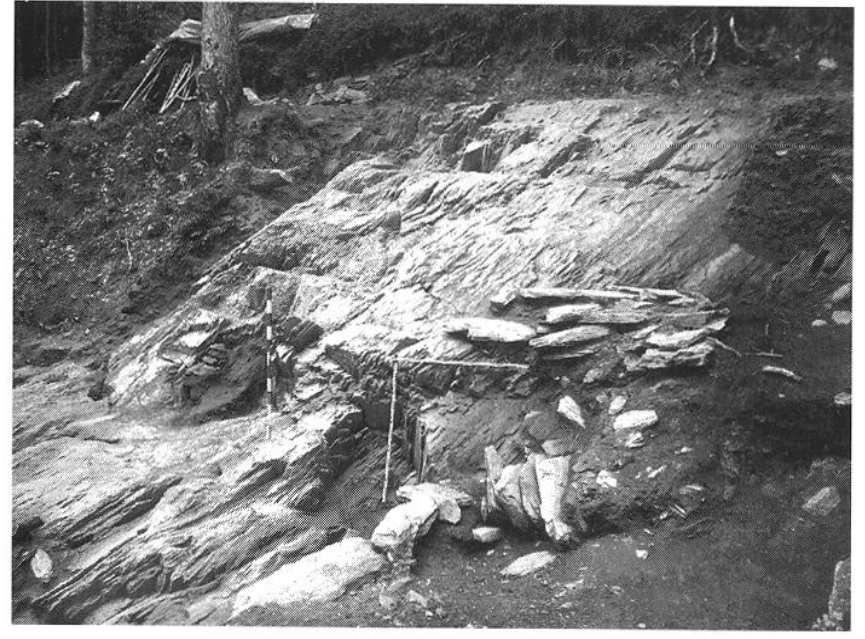

Fig. 21 - I.ercoul (Ariège) : ì gauche, le bas fourneau C isolé avec une partie de son remplissage, le jalon de 0,30 m est posé sur une entaille latérale, deux autres sont visibles juste au-dessus; au centre, le bas fourneau jumeau $B$; à droite, le vestige supérieur de la superstructure du bas fourneau jumeau A et une partie de l'éboulis écroulé dans le creuset.

creusée dans l'argile (fig. 20, $\mathrm{n}^{\circ}$ 3) et plusieurs trous de poteaux, dont un contenait un tronçon de branche de sapin. Elles recouvrent également l'éboulis provenant de l'effondrement du bas fourneau A, situé au nord (fig. 20, $n^{\circ} 5$ ). Les niveaux inférieurs, quant à eux, s'accumulent dans la pente, à l'est, et débordent à peine sur la plateforme (fig. 20, $n^{\text {os }} 4$ et $4^{\text {bis }}$ ).

Le bas fourneau A se présente sous la forme d'une cuve creusée dans le schiste (fig. $20, \mathrm{n}^{\circ} 7$ et fig. 22), ouverte vers l'est, de plan ovoïde $(0,80 \mathrm{~m}$ en est-ouest x $0,70 \mathrm{~m}$, à $0,60 \mathrm{~m}$ au-dessus du fond). Le fond de cuve offre un diamètre de $0,20 \mathrm{~m}$ et s'ouvre vers l'est sur un petit chenal, lui aussi taillé dans le socle rocheux, à profil en " V », large de $0,10 \mathrm{~m}$ pour $5 \mathrm{~cm}$ de profondeur. L'intérieur de cette cuve présente encore un morceau de revêtement interne en place. Il s'agit d'une argile cuite, rouge, bourrée de fragments millimétriques de schiste. Sa face interne est scoriacée. Le fond de la cuve et le secteur nord de la paroi sont plaqués d'une croûte de scorie. Le schiste du socle est rougi sur plusieurs dizaines de centimètres autour de la cuve. Il présente des entailles d'une dizaine de centimètres de largeur, symétriques de part et d'autre du creuset. Au-dessus de ce dernier, subsistent, sur une hauteur de $0,80 \mathrm{~m}$, du côté ouest, les vestiges d'une superstructure en moellons de schiste liés à l'argile,

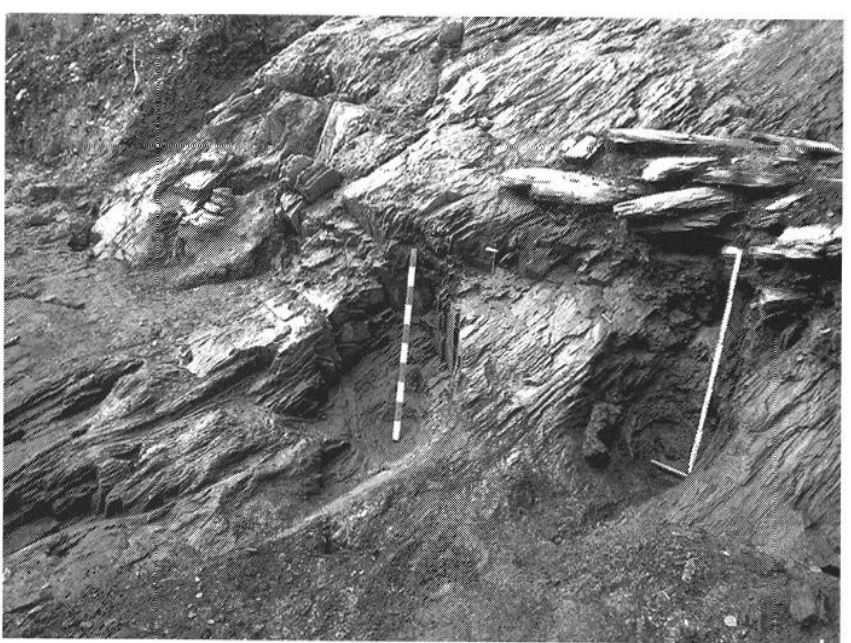

Fig. 22 - Lercoul (Ariège) : de droite à gauche, les trois bas fourneaux $(A, B$ et () .

posée sur le socle (fig. 21 à 23); son parement nord est rectiligne. Des empilements de blocs de schiste situés entre les cuves des fourneaux A et B étaient renversés vers le nord (fig. 21); il convient sans doute de les rattacher à la superstructure de ce premier fourneau. Un bloc de gneiss de $1 \mathrm{~m} \times 0,50 \mathrm{~m} \times 0,30 \mathrm{~m}$, présentant des traces de chauffe, était abattu entre les deux fourneaux (fig. 21 et 24). Redressé, il pourrait faire songer à une sorte de pied-droit, placé sur un côté de la porte du fourneau, semblable à ceux des bas fourneaux du $\mathrm{I}^{\mathrm{er}} \mathrm{s}$. avant notre ère aux Martys (Domergue dir., 1993). La présence de montants en pierre, monoblocs, destinés à supporter un linteau est aussi attestée dans certains bas fourneaux du haut Moyen Âge du canton de Vaud, en Suisse ${ }^{78}$.

La cuve du bas fourneau A n'est séparée de sa voisine, au sud (fourneau B), que par un éperon du socle de $0,70 \mathrm{~m}$ de largeur (fig. $20, \mathrm{n}^{\circ} 8$ et fig. 22). Ce deuxième creuset est identique au premier par sa forme, son chenal, ses entailles symétriques, mais il est un peu plus évasé. Il ne conserve qu'un petit bloc de scorie soudé au fond de la cuve et rien ne subsiste du revêtement intérieur, ni de la superstructure (fig. 21, 22 et 24).

78. P.-L. Pelet interprète en ce sens la présence d'un bloc erratique de $250 \mathrm{~kg}$ dans la construction des fours jumeaux des Bellaires III (Pelet, 1993, p. 55). C'est clairement le cas pour le bas fourneau I du site de Montcherand, fouillé par E. Abetel (1992, p. 19 et 24). 


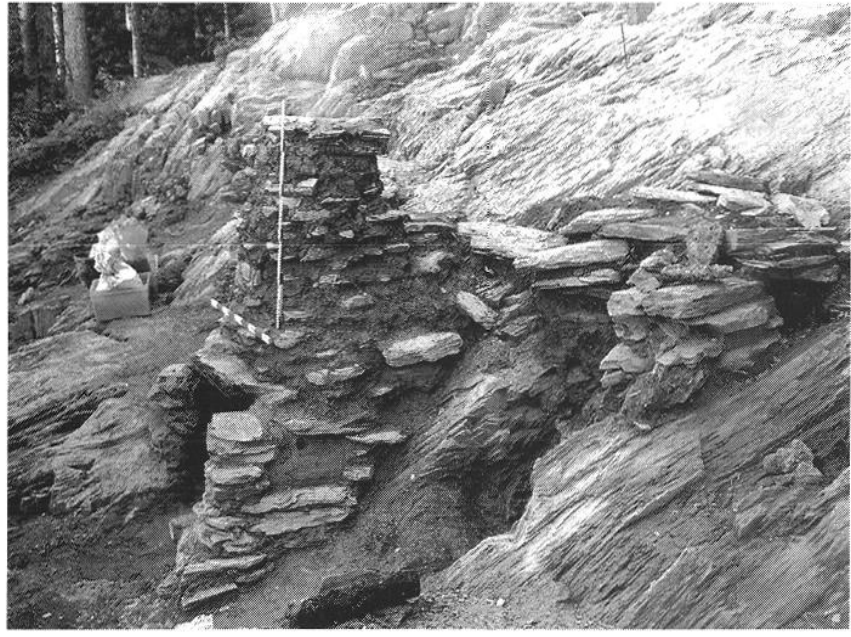

Fig. 23 - Lercoul (Aviegge) : au premier plan, la cuve et les vestiges de la cheminée du bas fourneau $A$; au second plan, avec des jalons de $1 \mathrm{~m}$, le bas fourneau expérimental construil sur le crensel archéologique du bas fourneau B (sept. 1999). À comparer avec les vestiges de la fig. 24.

Vers l'est, c'est-à-dire à l'avant des fourneaux, le socle présente un dénivelé d'environ $0,40 \mathrm{~m}$ sur une emprise de $2 \mathrm{~m}$, puis demeure horizontal sur $2 \mathrm{~m}$ de plus. Cette terrasse semble naturelle et devait être apparente dans la topographie de surface originelle, comme nous l'avons suggéré plus haut. La même rupture de pente s'observe au sud du fourneau $B$, mais à une cote plus élevée d'environ $0,50 \mathrm{~m}$ (fig. 22). La plate-forme d'argile présente elle aussi ce dénivelé de $0,40 \mathrm{~m}$ sous forme d'une marche allongée d'est en ouest (fig. 20).

Un ferrier occupait le centre et l'est du site (fig. 20, $\mathrm{n}^{\circ} 10$ ). Il était constitué de scories dont le détail et la typologie seront donnés plus loin, mais également, comme pour l'amas déjà décrit au nord, de lentilles et dépôts de charbons de bois, de plaques de schiste chauffées, etc. L'essentiel de ce ferrier se situait à l'est de la rupture de pente qui est soulignée, en face du fourneau $\mathrm{C}$, par une ligne de plaques de schiste plantées de chant dans l'argile. Le ferrier était formé par la superposition, sur $0,70 \mathrm{~m}$ de hauteur, de 7 sols indurés d'environ $1 \mathrm{~m}^{2}$, aux limites mal définies. Vers l'ouest, la couche de scories débordait sur la plate-forme, où elle n'avait que quelques centimètres d'épaisseur. Là, elle entourait et recouvrait partiellement deux trous de poteaux, un sol ferrugineux d'environ $1 \mathrm{~m}^{2}$ et l'éboulis du bas fourneau C. Le sol ferrugineux (fig. 20, $\mathrm{n}^{\circ} 11$ ), épais de $7 \mathrm{~cm}$, est constitué de microscories soudées. Au microscope ${ }^{79}$, on y décèle la

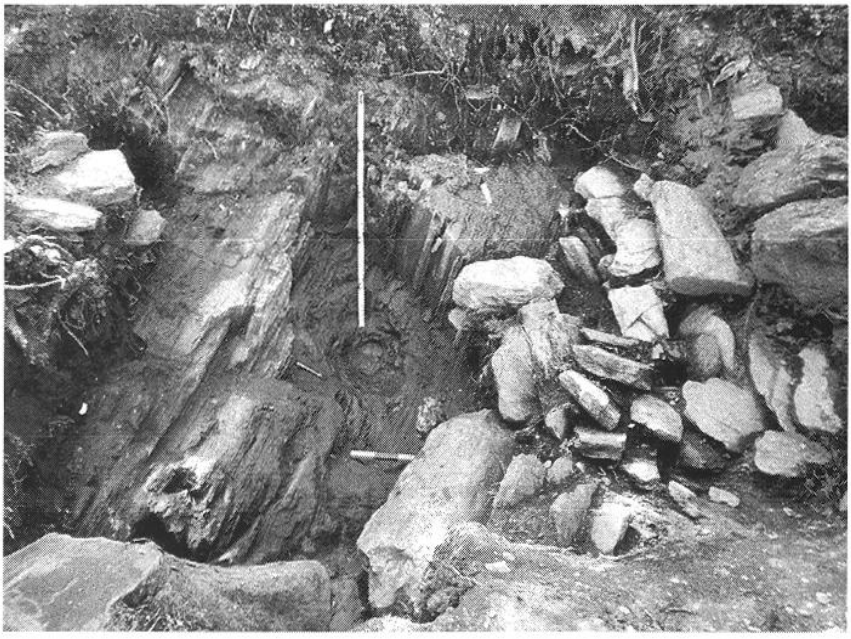

Fig. 24 - Lercoul (Ariege) : crenset du bas fourneau B (remarquer sur le bord gauche une entaille destinée à asseoir la superstructure) ; à droite, éboulis qui recoume le bas fourneau jumeau $A$; au centre et en bas, le bloc de gneiss couché pouvant être un pied-droit abattu.

présence de battitures typiques d'un travail d'épuration des massiaux. L'éboulis, constitué de blocs de schiste liés par un mortier d'argile très solide, était situé à 2 m à l'est du four $\mathrm{C}$ auquel il était relié par une nappe d'argile rouge contenlant des plaques de schiste et de grands blocs de revêtement réfractaire scorifié. Les scories qui recouvraient partiellement ces vestiges pouvaient provenir de l'affaissement d'un tas placé juste à l'est au sommet du ferrier. Ce secteur a été particulièrement perturbé par les racines d'un gros sapin.

L'ensemble de la plate-forme, du sud jusqu'à la rupture de niveaux signalée un peu plus haut, était jonché de plaques de schiste, pour quelques-unes chauffées, de scories et de parois de fours, sous une colluvion de pente. Ces débris reposaient sur un sol argileux rubéfié et constellé de charbons de bois.

Comme ses voisins, le fourneau $\mathrm{C}$ est creusé dans le socle schisteux (fig. $20, \mathrm{n}^{\circ} 9$, fig. 21 et 22 ). Le creuset présente un fond plat, sans chenal à l'avant, qui mesure $0,80 \mathrm{~m}$ d'est en ouest et $0,60 \mathrm{~m}$ de largeur. La paroi ouest, haute de $0,75 \mathrm{~m}$, est évasée. Du côté sud, près du bord de la cuve, le socle présente deux entailles étagées, creusées sur $0,40 \mathrm{~m}$ et $0,70 \mathrm{~m}$ de longueur. À l'ouest, juste au-

79. Examen effectuć par J.-C. I.eblanc, professeur de métallographie, Arts ct Mćticrs, Bordcaux, autcur d'un DEA sur la caractérisation des battitures archéologiques, que nous remercions. 
dessus du bord de la cuve, une autre entaille, large de $0,30 \mathrm{~m}$ à $0,40 \mathrm{~m}$, s'allonge sur $1,50 \mathrm{~m}$ du nord au sud. Le côté nord forme un redent de $0,30 \mathrm{~m}$ de hauteur. À $0,70 \mathrm{~m}$ au nord de la cuve, une cupule de $5 \mathrm{~cm}$ de diamètre est creusée dans le schiste. À $10 \mathrm{~cm}$ plus au nord, un petit replat de forme carrée $(10 \mathrm{~cm} \times 10 \mathrm{~cm}) \mathrm{a}$ été également aménagé. À une dizaine de centimètres à l'ouest du redent, de la cupule et du carré, une saignée au profil en " $\mathrm{V}$ " s'allonge sur $1 \mathrm{~m}$ du nord-ouest au sudest. En prolongeant cette orientation et sa pente, on aboutit à l'arrière du fourneau, à $0,70 \mathrm{~m}$ du fond. Deux trous de poteaux ont été creusés dans le socle rocheux, respectivement à $0,50 \mathrm{~m}$ et $1,20 \mathrm{~m}$ au sud de ce fourneau.

Le fond du fourneau C est tapissé, sur 0,12 m d'épaisseur, d'une couche de petits fragments de scories compactés, avec des éléments argileux cuits. À l'ouest, le revêtement réfractaire, épais de 5 à $10 \mathrm{~cm}$, est décollé de la roche ; l'argile est cuite, grise vers l'intérieur du four, blanche au centre et rouge vers le rocher. Entre cette argile et le socle, une argile de colluvion parcourue de racines s'est intercalée. Un amas argileux mêlé de scories et de charbons de bois est collé, sur $10 \mathrm{~cm}$, contre le revêtement ouest et sur le fond, où il s'étale sur $2 \mathrm{~cm}$ d'épaisseur. Au-dessus, du côté sud, à $5 \mathrm{~cm}$ de la paroi schisteuse, sont empilés trois blocs de calcaire dolomitique gris fortement érodés, peut-être par chauffage.

Une autre structure métallurgique se situe à $4 \mathrm{~m}$ au sud-est du fourneau C (fig. 20, $\mathrm{n}^{\circ}$ 12). Le socle schisteux a été taillé de manière à former un espace horizontal de plan ovale $(1,30 \mathrm{~m} \times 0,70 \mathrm{~m})$. La paroi ouest-sud-ouest est verticale. Le fond, très irrégulier à cause de la schistosité et de nombreuses diaclases, était couvert d'une couche de charbons de bois. Une plaque, constituée de petites scories, de particules de minerai de fer et de charbons de bois écrasés, est collée au sol, contre le bord nord. Sa taille est celle d'un pied humain et elle peut marquer un poste de travail. À l'ouest et en contre-haut de cette structure, le socle rocheux présente une rubéfaction due au chauffage. À moins de $1 \mathrm{~m}$ au sud-est de cette structure, deux trous de poteaux ont été creusés dans l'argile.

Une dizaine d'entailles, d'une surface de quelques dizaines de centimètres carrés à plus de $1 \mathrm{~m}^{2}$, sont taillées dans le socle, en amont de cette structure, sur $6 \mathrm{~m}$ de long en nord-sud (fig. 20). En outre, de nombreuses encoches émaillent le banc rocheux.

\section{COMBUSTIBLE, MINERAI ET SCORIES}

L'étude de 1350 charbons de bois provenant de prëlèvements effectués dans les différentes couches des ferriers a été réalisée. Les échantillons ont été obtenus par tamisage des sédiments à la maille de $5 \mathrm{~mm}$. La majorité de ce corpus (98\%) est constituée par le hêtre. Les $2 \%$ restants comportent du sapin, du buis et du sorbier. Ces résultats sont à mettre en rapport, d'un point de vue paléoenvironnemental, avec l'histoire de la sapinière locale, telle qu'elle a été résumée dans l'introduction de cet exposé ${ }^{80}$. La présence, même faible, du sapin est confirmée par le moignon de branche découvert dans l'un des trous de poteaux.

Le minerai récupéré dans les unités stratigraphiques du ferrier nord est constitué de gœthite, d'oligiste et de limonite. Ces oxydes sont typiques des formations supergènes des filons connus à Lercoul et à Rancié. Tous sont calibrés à la maille d'environ $2 \mathrm{~cm}$ et présentent des traces de chauffe. Un fragment de gros galet glaciaire en granite anatectique roux, de $0,15 \mathrm{~m}$ de côté en moyenne, dont deux faces sont martelées, semble être un morceau d'enclume ayant servi à concasser le minerai. Une analyse effectuée sur un échantillon de minerai trouvé en fouille indique des teneurs de $90,95 \%$ de $\mathrm{Fe}_{2} \mathrm{O}_{3}, 3,64 \%$ de $\mathrm{SiO}_{2}, 0,17 \% \mathrm{~d}^{\prime} \mathrm{Al}_{2} \mathrm{O}_{3}$ et $2,29 \%$ de $\mathrm{MnO}^{81}$. La forte teneur en manganèse est typique des minerais locaux ${ }^{82}$. En 1868, l'ingénieur des Mines Jean Mussy, en poste à Vicdessos, publiait les analyses de

80. La fouille programmée de Lercoul a concerné le site sidérurgique, objet de cet article, mais également la forêt charbonnée du versant, avec la fouille d'une dizaine d'emplacements de charbonnage, souvent avec des reprises et abandons successifs, datés du V* au XVII s. et couvrant tout le Moyen Âge. Voir C. Dubois, L'archéologie au service de la science forestière, Arborescences, 71, dossier Forệ et archéologie, Paris, éd. Office national des forêts, nov.-déc. 1997, p. 15-16 et C. Dubois, J.-P. Metailié et V. Izard, Archéologie de la forêt charbonnée : questions et méthodes, illustrées par l'exemple du site de I.ercoul (Ariège), in: La dynamique des paysages protohistoriques, antiques, médiévaux et modernes, Actes des XVII rencontres internationales d'archéologie et d'histoire d'Antibes, 19-21 oct. 1996, Sophia Antipolis, éd. APDCA, 1997, p. $525-540$.

81. Analyse CRPG du CNRS, Nancy, sous la direction de A. Ploquin.

82. Une sćrie d'analyses effectuée sur des prises d'essai d'hématite brune, par l'ingénieur des Mines en poste à Vicdessos de 1877 à 1881, donnait des teneurs de $52,85 \%$ de fer et $4,13 \%$ de manganèse rapportées au minerai sec. (A. Carcanagues, Étude sur la tencur en fer et en manganèse des minerais de Rancié (Ariège), Annales des Mines, $8^{c}$ série, 5, 1884, p. 115-122). 
60 échantillons prélevés dans les divers chantiers de Rancié $^{83}$. Vingt-trois d'entre eux concernent les niveaux supérieurs de la mine, semblables aux filons de Lercoul et excluent tout carbonate. Le choix d'échantillons par l'ingénieur est peut-être contestable du point de vue de la représentativité statistique de l'ensemble, mais sans doute proche de la pratique des mineurs et métallurgistes du III ${ }^{\mathrm{e}} \mathrm{s}$. Les teneurs moyennes sur ces 23 échantillons sont les suivantes : $75,78 \%$ de $\mathrm{Fe}_{2} \mathrm{O}_{3}$, soit $52,7 \%$ de $\mathrm{Fe}, 4,16 \%$ de $\mathrm{MnO}$, soit $3,2 \%$ de $\mathrm{Mn}$, et $8,1 \%$ de Si.

Un échantillon de $2 \mathrm{~m}^{3} \mathrm{du}$ ferrier a été trié manuellement: toutes les scories ayant au moins 8 à $10 \mathrm{~cm}$ de côté ont été mises à part et retriées par types. Les scories non coulées et les fonds de four représentent $81,6 \%$ du total, les scories coulées $9 \%$, les argiles scorifiées 7,8\% et les scories non coulées très denses $1,6 \%$. Un autre tri, effectué sur un tiers de mètre cube, en tenant compte du gravier de scories, permet de relever le taux des scories coulées à $14 \%$ du total. Des analyses de ces différents matériaux, d'un échantillon de minerai trouvé en fouille et d'une plaque de schiste, ont été faites ${ }^{84}$. Les résultats montrent d'intenses échanges entre le revêtement intérieur des fours et la scorie. Sept échantillons de scories s'avèrent fortement contaminés par les parois; ils contiennent, en moyenne, $47,56 \%$ de $\mathrm{SiO}_{2}, 10,33 \%$ de $\mathrm{Al}_{2} \mathrm{O}_{3}, 31,86 \%$ de $\mathrm{Fe}_{2} \mathrm{O}_{3}$ et $3,19 \%$ de MnO. En comparaison, neuf autres renferment $18,11 \%$ de $\mathrm{SiO}_{2}, 2,93 \%$ de $\mathrm{Al}_{2} \mathrm{O}_{3}, 62,26 \%$ de $\mathrm{Fe}_{2} \mathrm{O}_{3}$ total et $5,68 \%$ de $\mathrm{MnO}$. L'examen des divers diagrammes et les analyses de terres rares soulignent cette partition des scories et ce phénomène d'intense contamination. Le manganèse semble être surtout passé dans les scories, ce qui pourrait signifier que le fer métallique produit était relativement doux. D'autres analyses permettent de déterminer les températures maximales obtenues, soit $1300 / 1350{ }^{\circ} \mathrm{C}$ (Jarrier, 1993, p. 65-79).

Les ferriers renfermaient d'autres pièces intéressantes: quelques fragments de tuyères et des morceaux de pains d'argile scoriacés. Sur ces deux types d'objets, des empreintes de végétaux (peut-être d'aiguilles de sapin) sont visibles.

83. J. Mussy, Description de la constitution géologique et des ressources minérales du canton de Vicdessos et spécialement de la mine de Rancié, Annales des Mines, 6" série, XIV, 1868, p. 284-287.

84. Idem note 81.

\section{COMMENTAIRES}

Il est clair que, des deux fours jumeaux, seul le fourneau A était pourvu d'une superstructure au moment de son abandon : elle s'est écroulée et répandue sur le sol de travail. Le fourneau $A$ a donc fonctionné le dernier : ou bien il est postérieur à $\mathrm{B}$, ou bien $\mathrm{A}$ et $\mathrm{B}$ ont fonctionné alternativement, en batterie, jusqu'au moment où $\mathrm{A}$ fut seul maintenu en service. Dans un cas comme dans l'autre, pour quelle raison le fourneau $B$ fut-il abandonné ? Peut-être son profil évasé traduit-il une usure devenue incompatible avec un bon fonctionnement?

Nous verrons plus loin, à propos des datations, que la vidange de ces deux fourneaux s'effectuait vers le nordest. La présence des vestiges de la superstructure du four A permet de restituer un massif de plan carré formant une cheminée au-dessus du creuset (fig. 21 et 24); l'ensemble ayant une hauteur conservée et minimale de $1,40 \mathrm{~m}$. D'autre part, la position stratigraphique de l'éboulis de la superstructure, partiellement couvert de déchets métallurgiques, indique que l'activité sidérurgique s'est poursuivie après l'abandon et la ruine de ce bas fourneau.

Or, dans le secteur méridional du site, à un niveau légèrement supérieur, se trouve le fourneau $C$. De prime abord son aspect le différencie de $\mathrm{A}$ et de $\mathrm{B}$. Mais il y a plusieurs points communs, en particulier l'encastrement dans le socle rocheux et les entailles périphériques (fig. 21 et 22), vraisemblablement destinés à asseoir les assises de la superstructure. De plus, les parois scoriacées découvertes contre le côté ouest sont semblables à celles du bas fourneau A et à celles trouvées dans les ferriers. Les différences tiennent surtout à sa plus grande taille, près du double, à son fond plat et à la présence des blocs de dolomie. La plus grande capacité n'est pas aberrante, si l'on songe aux bas fourneaux des Martys, par exemple. Elle pourrait même constituer un progrès. D'ailleurs l'éboulis correspondant à l'effondrement de la superstructure laisse penser que cette dernière était imposante. Quant aux blocs de dolomie qui semblent vouloir rétrécir le creuset, nous ignorons s'ils étaient censés jouer un rôle dans l'opération de réduction directe, ou bien s'il s'agit d'un témoin d'un usage postérieur et différent de ce four. Les fonctions du redent, de la saignée, de la cupule et du carré nous échappent: s'agit-il d'aménagements liés à l'installation d'un soufflet ? En tout cas, bien que nous ne puissions tout expliquer de manière satisfaisante, il 
semble que le fourneau $\mathrm{C}$ soit bien un bas fourneau, stratigraphiquement postérieur aux fourneaux A et B.

Qu'il s'agisse des deux fours jumeaux A et B ou du fourneau $C$, il est probable que la façade orientale de la superstructure devait être close par une paroi faite de pains d'argile réfractaire, cassée en fin de réduction. En ce qui concerne $\mathrm{A}$ et $\mathrm{B}$, la porte devait être percée d'un orifice à sa base, correspondant au chenal taillé dans le socle. Par là coulait la scorie qui se répandait en éventail sur le sol rocheux. La racine d'un éventail de ce genre montre que le diamètre de ce trou devait mesurer $5 \mathrm{~cm}$. Les rares fragments de tuyères attestent l'emploi d'une ventilation artificielle, ce que la température atteinte suggérait déjà. Tuyères, pains d'argile et revêtement interne des creusets étaient faits d'argile et de schiste pilé, d'origine locale.

La structure ovale méridionale est un aménagement lié à une combustion (fig. $20, \mathrm{n}^{\circ} 12$ ); les charbons de bois présents au fond en témoignent. La rubéfaction du schiste à l'ouest et en contre-haut suggère un flux de chaleur dont l'origine se situerait vers les deux trous de poteaux, lesquels peuvent signaler l'emplacement d'un soufflet. Deux hypothèses sont possibles. Les fragments de minerai découverts dans le secteur nord du site portent des traces de chauffe indiquant que le minerai était calciné, préalablement à son concassage et à son introduction dans les bas fourneaux. Un feu de branchages suffit à cette opération, qui a pour but de déshydrater les oxydes. Mais nous n'avons pas découvert de tels fragments aux alentours de la structure. L'autre hypothèse est qu'à cet emplacement l'on réchauffait le massiau en vue de son épuration. Cette dernière opération semble avoir été effectuée à $3 \mathrm{~m}$ au nord, comme en témoigne la présence de battitures.

L'ensemble des trous de poteaux mis au jour ne dessine pas un plan régulier (fig. 20). Tous ne sont pas contemporains, tous n'avaient certainement pas le même usage. Quelques-uns ont dû soutenir une toiture destinée à protéger des intempéries le minerai calciné, le charbon de bois et les hommes. Des traverses pouvaient s'appuyer sur la paroi rocheuse, dans les multiples entailles dont elle est creusée. Nous n'avons découvert aucun vestige de couverture. Une toiture en tuiles ou en lauzes de schiste aurait nécessité une véritable charpente. Nous penchons pour une couverture en matière végétale, périssable, facilement réparable au début de chaque nouvelle saison d'activité sidérurgique.

\section{DATATION, CHRONOLOGIE}

À l'exception de deux petits tessons de céramique commune à pâte sombre recueillis lors du tamisage d'une couche de scories au sommet du ferrier dans la partie nord du site, les seuls fragments de céramique découverts l'ont été dans les sondages de reconnaissance effectués en 1990, dans la pente sous la plateforme. Le sondage 2 a livré, parmi les scories, cinq tessons de vaisselle commune cuite en atmosphère oxydante. Quatre ont des pâtes siliceuses riches en mica et sont atypiques. Le cinquième présente un dégraissant sombre et un lissage de surface qui évoque certaines poteries communes claires d'époque gallo-romaine. Dans le sondage 3 a été découvert un fond de récipient (diam. : $10 \mathrm{~cm}$ ), plat, à pâte noire et dégraissant de mica, qui pourrait se rattacher à la typologie régionale du haut Moyen Âge.

La pauvreté du mobilier exhumé et son imprécision chronologique nous ont conduit à avoir recours aux datations ${ }^{14} \mathrm{C}$. Toutes nos datations proviennent d'échantillons d'une vingtaine de grammes chacun, tous constitués uniquement de hêtre en branchettes ou en bois ne comptant que quelques cernes jusqu'au dcrnier avant l'écorce. Les quantités données au laboratoire permettent de limiter l'incertitude à \pm 40 ans et le choix de bois jeune assure une date proche de l'abattage.

Dans le sondage 2 de 1990, dans la pente, au nord et sous la plate-forme, nous avions prélevé un échantillon (1) dans le ferrier. L'âge brut est $1690 \pm 50 \mathrm{BP}$ avec une date calibrée de 180-530 cal. AD (Archéolabs, ARC 514). Lors de la première campagne de fouille, en 1995, un nouvel échantillon (2) a été prélevé à la base du ferrier, sur la plate-forme, en face du fourneau C. L'âge brut donne $1715 \pm 40 \mathrm{BP}$ et la date calibrée est $180-420 \mathrm{cal}$. AD (Archéolabs, ARC 1375). En 1997, nous avons fait dater un lot de charbons (3) provenant du petit dépôt situé dans un recoin de la paroi rocheuse, au sommet de la stratigraphie (fig. $20, \mathrm{n}^{\circ} 1$ ). L'âge brut obtenu est de $1720 \pm 40 \mathrm{BP}$ pour une calibration de $180-415 \mathrm{cal}$. AD (Archéolabs, ARC 1702). Enfin, des charbons (4) provenant d'une couche de déchets de métallurgie située stratigraphiquement sous la précédente ont été également soumis à l'analyse radiocarbone. Leur âge brut est plus ancien : $1830 \pm 40 \mathrm{BP}$, ainsi que les dates calibrées : 70-320 cal. AD (Archéolabs, ARC 1701). 
Il faut examiner ces datations en fonction de la situation des échantillons en plan et en stratigraphie. Comme on l'a déjà vu, la disposition des éboulis des fourneaux et des ferriers indique que les deux fours jumeaux A et B fonctionnèrent antérieurement au fourneau $\mathrm{C}$. Dans la première phase, les scories et autres déchets auraient été versés au nord, jusqu'à la couche du prélèvement (4). Les scories et charbons de la seconde phase se seraient entassés à l'est (deuxième échantillon), au sud, mais aussi au nord (échantillon 3), où ils recouvrent des vestiges de la phase antérieure. Ce scénario explique logiquement les datations 2, 3 et 4 . Mais que dire de la première ? Le sondage 2 a été implanté assez bas sur la pente, peut-être à un niveau où auraient roulé les déchets les plus récents. Ou bien, les conditions du sondage étant difficiles (étroitesse, front ébouleux), l'échantillonnage a été défectueux.

Compte tenu de cette hypothèse de deux phases chronologiquement distinctes, nous pouvons retenir, pour la première, les dates calibrées $70-320 \mathrm{cal}$. $\mathrm{AD}$, sachant qu'il y a un pic de probabilité de $90,7 \%$ pour l'intervalle 70-255 cal. AD. Pour la seconde phase, un calcul de moyenne pondérée, effectué par Archéolabs, donne un âge ${ }^{14} \mathrm{C}$ conventionnel de $1710 \pm 24 \mathrm{BP}$, et une date calibrée située entre 255 et 405 cal. $\mathrm{AD}$. Étant donné que la stratigraphie ne révèle pas d'hiatus tel que le matérialiserait un niveau de colluvion de pente entre l'éboulis du bas fourneau A et les scories qui le recouvrent, il est probable que les deux phases se sont succédé rapidement, certainement autour du milieu du III ${ }^{\mathrm{e}} \mathrm{s}$.

\section{ESTIMATIONS CHIFFRÉES}

S'agissant des ferriers, d'après les hauteurs des stratigraphies et les mesures de surface, ce sont environ $500 \mathrm{~m}^{3}$ de déchets et de matrice terreuse qui ont été rejetés, soit a peu près $300 \mathrm{~m}^{3}$ de scories. Les mesures de densité effectuées lors de nos tris sur ces $300 \mathrm{~m}^{3}$ de scories, scories en menus graviers comprises, donnent un chiffre moyen de $1,25 \mathrm{t} / \mathrm{m}^{3}$. Les scories produites pèseraient ainsi $375 \mathrm{t}$, que nous arrondissons à $400 \mathrm{t}$.

Pour estimer grossièrement les quantités de minerai consommé et de fer produit, ainsi que la durée d'activité du site, nous nous appuyons sur une donnée expérimentale. En septembre 1999, nous avons reconstitué et utilisé un bas fourneau sur les vestiges du creuset archéo- logique $B^{85}$ (fig. 23). Seuls le schiste et l'argile du site ont été employés dans la construction et le revêtement interne. Nous avons grillé puis concassé à la maille de $2 \mathrm{~cm}$ du minerai de fer local, hématite et gœthite, en tout point semblable à celui utilisé au $\operatorname{III}^{e} s$. Notre combustible pour les réductions était du charbon de bois de hêtre local, fabriqué en meule. La première réduction et la troisième ont été défaillantes, en raison de défauts de température. En revanche la seconde opération a été positive : à partir de $30 \mathrm{~kg}$ de minerai grillé et de $34,5 \mathrm{~kg}$ de charbons de bois, nous avons obtenu un massiau de fer de $6 \mathrm{~kg}$ et $17 \mathrm{~kg}$ de déchets de tous types (scories coulées, scories de parois, argile cuite e.t arrachée au défournement). Considérant que notre savoir-faire est bien inférieur à celui des métallurgistes qui conduisaient ces bas fourneaux du $\mathrm{III}^{\mathrm{e}}$ s., nous tenons nos chiffres comme une base de calcul minimale. Les anciens sidérurgistes de Lercoul passaient vraisemblablement davantage de minerai à chaque opération, obtenaient une quantité de fer plus importante et proportionnellement certainement moins de déchets. La grande taille $\mathrm{du}$ bas fourneau $\mathrm{C}$ améliorait vraisemblablement ces paramètres.

À partir de l'expérimentation réussie, on retient que le poids du minerai traité égale le poids des déchets multiplié par 1,75. Le poids du charbon de bois consommé, chauffage du bas fourneau inclus, est le double de celui des scories. Ainsi les $400 \mathrm{t}$ de scories proviendraient de la réduction d'au moins $700 \mathrm{t}$ de minerai grillé. Notre rendement en fer produit, qui correspond à $20 \%$ du minerai de départ, donnerait $140 \mathrm{t}$ de fer issues des bas fourneaux, au minimum. La consommation globale de charbons de bois pourrait avoir atteint $800 \mathrm{t}$. À raison d'environ $9 \mathrm{~m}^{3}$ de hêtre vert pour $1 \mathrm{t}$ de charbons de bois, ces 800 t représentent 7200 stères de hêtre, soit encore 90000 hêtres de taillis de $10 \mathrm{~cm}$ de diamètre et $10 \mathrm{~m}$ de hauteur.

À raison de $17 \mathrm{~kg}$ de scories par opération, les $400 \mathrm{t}$ $\mathrm{du}$ site correspondraient à 23530 réductions. Si l'on suppose un rythme de 25 opérations par mois pour une saison de 6 ou 7 mois par an, on aboutit à une durée d'activité totale de 156 à 134 ans. Dans la mesure où ce résultat est lié à notre rendement expérimental, il est probable, vu le savoir-faire des anciens métal-

85. Expćrimcntations de réductions conduites par J. C. Leblanc, membre associé de l'UMR 5608, UTAH, que nous remercions vivement. 
lurgistes, que la durée d'activité réelle du site ait été plus courte.

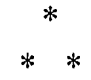

En Europe, les bas fourneaux dont la cuve est semiencastrée dans un talus argileux ou une moraine sont nombreux. À notre connaissance, du moins pour la France et les pays voisins, les fours de Lercoul constituent le premier exemple d'encastrement dans le socle rocheux du substrat. La nature de celui-ci, du schiste facile à creuser, s'y prêtait bien et le relief invitait à un tel type d'installation. Le chenal d'évacuation des scories coulées, sans être tout à fait original, est assez rare. Nous n'en avons trouvé qu'un seul autre exemple, à Frouard en Lorraine, où ce chenal est creusé dans l'argile (Leroy, 1997, p. 121). Avec leur superstructure en moellons de schiste qui formait cheminée, ces appareils atteignaient au minimum $1,40 \mathrm{~m}$ de hauteur et vraisemblablement davantage. Le minerai de fer local, principalement gœthite et oligiste riches en manganèse, était calciné, mis à la maille de $2 \mathrm{~cm}$ et réduit avec du charbon de bois de hêtre, à une température d'environ $1300{ }^{\circ} \mathrm{C}$. Les massiaux étaient épurés sur place.

Deux générations de fourneaux se sont succédé : les deux bas fourneaux jumeaux $\mathrm{A}$ et $\mathrm{B}$ au nord de la plateforme, puis le fourneau $C$ situé plus au sud, plus grand et doté d'aménagements externes et internes que nous comprenons mal. Cette succession s'est probablement déroulée vers le milieu du III' $s$. de notre ère. En raison de l'indigence du site en mobilier daté, cette conclusion est basée sur quatre datations au radiocarbone et la chronologie relative s'appuie sur l'analyse stratigraphique.

D'après une expérimentation archéologique réussie, mais dont les données quantitatives ne sauraient constituer que des valeurs minimales par rapport à la réalité, le ferrier de $400 \mathrm{t}$ correspondrait à des consommations de $800 \mathrm{t}$ de charbon de bois et $700 \mathrm{t}$ de minerai grillé, et à une production de $140 \mathrm{t}$ de fer. 